
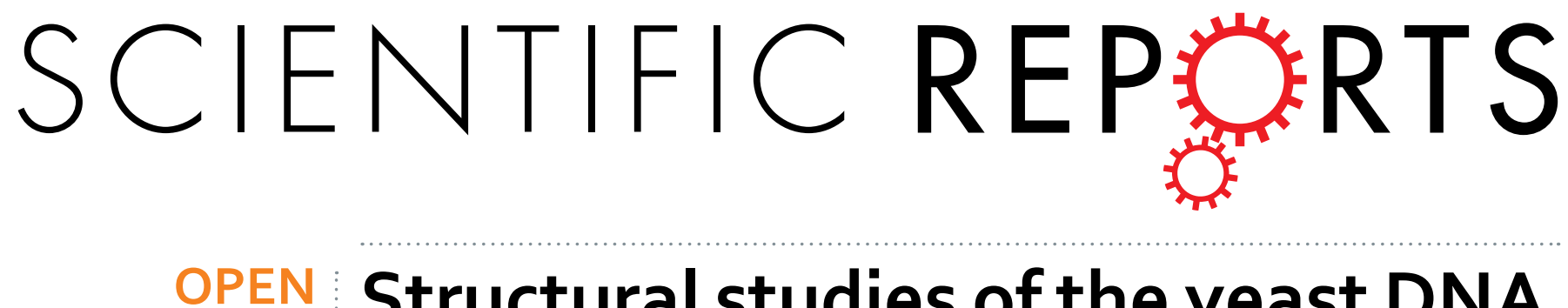

\title{
Structural studies of the yeast DNA damage-inducible protein Ddi1 reveal domain architecture of this \\ eukaryotic protein family
}

Received: 28 June 2015

Accepted: 01 September 2016

Published: 20 September 2016
Jean-François Trempe ${ }^{1,+}$, Klára Grantz Šašková ${ }^{2,3}$, Monika Siváa ${ }^{2,3,4}$, Colin D. H. Ratcliffe $^{1}$, Václav Veverka ${ }^{2}$, Annabelle Hoegl ${ }^{1}$, Marie Ménade ${ }^{1}$, Xin Feng ${ }^{1}$, Solomon Shenker ${ }^{1}$, Michal Svoboda ${ }^{2,5}$, Milan Kožíšek ${ }^{2}$, Jan Konvalinka ${ }^{2,3}$ \& Kalle Gehring ${ }^{1}$

The eukaryotic Ddi1 family is defined by a conserved retroviral aspartyl protease-like (RVP) domain found in association with a ubiquitin-like (UBL) domain. Ddi1 from Saccharomyces cerevisiae additionally contains a ubiquitin-associated (UBA) domain. The substrate specificity and role of the protease domain in the biological functions of the Ddi family remain unclear. Yeast Ddi1 has been implicated in the regulation of cell cycle progression, DNA-damage repair, and exocytosis. Here, we investigated the multi-domain structure of yeast Ddi1 using X-ray crystallography, nuclear magnetic resonance, and small-angle X-ray scattering. The crystal structure of the RVP domain sheds light on a putative substrate recognition site involving a conserved loop. Isothermal titration calorimetry confirms that both UBL and UBA domains bind ubiquitin, and that Ddi1 binds K48-linked diubiquitin with enhanced affinity. The solution NMR structure of a helical domain that precedes the protease displays tertiary structure similarity to DNA-binding domains from transcription regulators. Our structural studies suggest that the helical domain could serve as a landing platform for substrates in conjunction with attached ubiquitin chains binding to the UBL and UBA domains.

The ubiquitin system is primarily a signaling pathway whereby substrates tagged with various types of ubiquitin chains or ubiquitin-like (UBL) modifiers undergo different fates in the cell ${ }^{1}$. Ubiquitinated substrates are recognized by receptor proteins that contain ubiquitin-binding domains such as ubiquitin-interacting motifs (UIM) and ubiquitin-associated (UBA) domains ${ }^{2}$. In Saccharomyces cerevisiae, three ubiquitin receptors (Ddi1, Rad23, and Dsk2) have C-terminal UBA domains that bind ubiquitin and Lys48-linked polyubiquitin ${ }^{3-6}$. These proteins also bear an N-terminal UBL domain that binds Rpn1 in the 19 S proteasome subunit ${ }^{7-10}$. Ddil and Rad23 are DNA-damage inducible proteins, and both have been shown to suppress the temperature sensitivity of a $p d s 1$ mutant ${ }^{11}$. The protein Pds1 (securin) is a mitotic checkpoint control protein, and its ubiquitination by the anaphase-promoting complex (APC) and subsequent degradation is required for the separation of sister chromatids. The triple-deletion mutant $\Delta d d i 1 \Delta \mathrm{rad} 23 \Delta d s k 2$ shows a synthetic effect and delays in the onset of G2/M phase and anaphase, suggesting redundant roles in cell cycle progression ${ }^{12}$.

Over the last ten years, the biology of yeast Ddil has been investigated from different perspectives. The expression of the DDI1 gene is controlled by a bidirectional DNA-damage inducible promoter that divergently transcribes DDI1 and MAG1, a 3-methyladenine DNA glycosylase involved in a base-excision-repair pathway ${ }^{13,14}$. These two genes are differentially regulated in response to different DNA-damage checkpoint pathways ${ }^{15-17}$.

${ }^{1}$ Groupe de Recherche Axé sur la Structure des Protéines, Department of Biochemistry, McGill University, 3649 Promenade Sir William Osler, Montreal, OC, H3G OB1, Canada. ${ }^{2}$ Gilead Sciences and IOCB Research Center, Institute of Organic Chemistry and Biochemistry of the Academy of Sciences of the Czech Republic, Flemingovo n. 2, 16610 Prague 6, Czech Republic. ${ }^{3}$ Department of Biochemistry, Faculty of Science, Charles University, Hlavova 8, 12000 Prague 2, Czech Republic. "First Faculty of Medicine, Charles University in Prague, Katerinska 32, 121 08, Prague 2, Czech Republic. ${ }^{5}$ Department of Physical and Macromolecular Chemistry, Faculty of Science, Charles University, Hlavova 8, 12000 Prague 2, Czech Republic. 'Present address: Department of Pharmacology \& Therapeutics, McGill University, 3655 Promenade Sir William Osler, Montreal, QC, H3G 1Y6, Canada. Correspondence and requests for materials should be addressed to J.-F.T. (email: jeanfrancois.trempe@mcgill.ca) 
Strong expression of MAG1 and DDI1 can thus be induced by the addition of methyl methane-sulfonate to yeast cells, which triggers the CHK1- and MEC1-dependent DNA-damage response (DDR) pathways. Recent studies also indicate a possible role for Ddil in degradation of the Ho endonuclease, the enzyme responsible for switching alleles at the mating type locus $M A T^{18}$. Activation of the $M E C 1$-dependent DDR pathway leads to the phosphorylation and rapid degradation of the Ho protein by the ubiquitin-proteasome system ${ }^{19}$. Phosphorylated nuclear Ho is exported to the cytoplasm via the Msn5 nuclear exportin and ubiquitinated by the SCFUfo1 E3 ligase $\operatorname{complex}^{20}$. Interestingly, Ho accumulates in $\Delta d d i 1$ cells, but not in $\Delta \mathrm{rad} 23$ or $\Delta d s k 2$ cells $^{18}$. This specificity was attributed to specific interactions between the UBL domain of Ddi1 and four tandem UIMs located at the C-terminus of Ufo ${ }^{21}$. Ufo1 binds phosphorylated Ho through its F-box domain to mediate SCF-dependent ubiquitination ${ }^{18}$.

Ddil (also known as Vsm1 from v-SNARE-master 1) was independently identified as a SNARE-interacting protein in a yeast two-hybrid screen using the endocytic Snc2 protein as a bait ${ }^{22}$. Ddil interacts with both exoand endocytic v-SNARE proteins (Snc1 and Snc2). Overexpression of Ddil in yeast bearing a mutation in the sec 9 gene ( $t$-SNARE) inhibits protein secretion, suggesting that Ddil is a negative regulator of exocytosis. It was later shown that Ddil binds to the exocytic t-SNARE Sso1, which precludes binding of Ssol to its functional partner Sec9 and thus inhibits exocytosis ${ }^{23}$. Binding of Ddil to Sso1 is promoted by phosphorylation of the $\mathrm{N}$-terminal autoinhibitory domain of Sso1. The interaction is mediated by a linker region of Ddil located between the protease and UBA domains. The linker includes a PEST motif and a phosphorylation site (T348) that also regulates exocytosis ${ }^{24}$. Consistent with these findings, $\Delta d d i 1$ yeast cells show increased global protein secretion ${ }^{23,25}$. Ddi1 is also required for endocytosis of the guanine nucleotide-binding protein $\mathrm{G}^{26}$. Overall, these various studies point towards a role for Ddil in cell cycle and growth control, as well as protein trafficking.

Yeast Ddil has three structural domains. It has an N-terminal UBL domain that shares only $14 \%$ sequence identity with ubiquitin. Its central retroviral protease-like domain (RVP), which is common to all eukaryotic Ddil orthologs, is homologous to retroviral aspartic proteases ${ }^{27}$. The active site aspartate is required for repression of protein secretion in yeast ${ }^{25}$, and this phenotype can be inhibited by HIV protease inhibitors ${ }^{28}$, strongly suggesting that this function of Ddil is linked to its protease activity. The Ddil-like protein from Leishmania major displays proteolytic activity at acidic $\mathrm{pH}^{29}$, suggesting that the protein may be active only in acidic vesicular compartments. The three-dimensional structure of the isolated protease domain confirms that the domain adopts the typical aspartyl protease fold, with a two-fold dyad symmetry that allows Asp220 from two subunits to form hydrogen bonds with a catalytic water molecule ${ }^{30}$. Yeast Ddil also bears a C-terminal UBA domain that is found only in plants, fungi, and invertebrates, but not in vertebrate Ddil orthologs ${ }^{3-5}$. The UBA domain from the Schizosaccharomyces pombe ortholog Mud1 binds selectively to K48-linked diubiquitin $\left(\mathrm{Ub}_{2}\right)$ through two ubiquitin-binding sites ${ }^{5}$. In spite of all these studies, the overall function and substrate(s) of Ddil protease domain remain elusive.

Here, we report findings from structural and functional studies of full-length Ddi1 from S. cerevisiae. We determined a new crystal structure of the RVP domain of Ddil that provides insight into its putative substrate recognition mechanism. We determined the solution structure of the UBL domain by NMR spectroscopy and performed interaction studies with different proposed ligands. We found that UBA and UBL both bind ubiquitin and that Ddil binds K48-linked diubiquitin with enhanced affinity. We also determined the structure of a new $\alpha$-helical domain (named HDD) that precedes the RVP domain and could play a role in substrate recognition. We used SAXS to investigate the structure and dynamics of the module formed by the UBL, HDD and RVP domains. Finally, we performed Proteomic Identification of protease Cleavage Sites (PICS) analysis with full-length Ddi1 at both acidic and neutral $\mathrm{pH}$ to explore substrate specificity ${ }^{31}$.

\section{Results}

Crystal structure of the Ddi1 retroviral protease-like domain reveals a potential substrate-binding loop. The most conserved segment of the Ddil family is its RVP domain, which probably defines its biochemical function. While the structure of the RVP had previously been determined ${ }^{30}$, the substrate-binding mode remained unknown. We obtained a new crystal structure of the RVP domain of Ddil (residues 185-325) at $1.8 \AA$ resolution. The unit cell dimensions differ from those of the previously published structure. The structure was solved by molecular replacement and refined to $R_{\text {work }} / R_{\text {free }}$ of $18.3 / 21.3 \%$ (Supplementary Table 1 ). The asymmetric unit consists of two chains, which form a dimer with non-crystallographic $\mathrm{C} 2$ symmetry. The structure is very similar overall to the previous one, with a backbone rmsd of $0.6 \AA$ for residues that are common to both structures. In the new structure, electron density is visible for the $\mathrm{N}$-terminal segment spanning residues 185-199. Intriguingly, this N-terminal segment binds into the active site (Asp220) of an adjacent protease dimer in a different asymmetric unit (Fig. 1a). The segment is positioned such that the active site Asp220 might cleave between amino acids 189 and 190 (Fig. 1b). However, the protein does not undergo auto-proteolysis in solution; mass spectrometry confirmed that the RVP domain remains intact even under acidic conditions (Supplementary Fig. S1). Nonetheless, while the observed arrangement is a likely an artefact of crystallization, the N-terminal segment acts as pseudo-substrate and reveals how Ddil might engage a substrate. The $\mathrm{N}$-terminus adopts a $\beta$-strand conformation that interacts extensively with a loop formed by residues $245-258$ (Fig. 1b). This loop is only visible in the chain that docks the N-terminus. There are multiple hydrogen bonds between the backbone atoms of the pseudo-substrate and the loop, suggesting that it might be involved in positioning the substrate for catalysis. The side chain of the conserved Gln224 (Fig. 1c) also makes a hydrogen bond with the backbone amide of the pseudo-substrate (Fig. 1b). The side chain of Phe246, which is conserved as an aromatic residue across Ddil orthologs, interacts with a methionine in the N-terminal fragment (Fig. 1c), suggesting this may be a specificity determinant for the protease activity of Ddil. Arg251 is also conserved and could potentially interact with a substrate, but we did not observe any electron density for its side chain, implying that it is disordered. Finally, the side chain of Ile191 fits snugly into a hydrophobic groove formed by the loop and the rest of the RVP domain. 


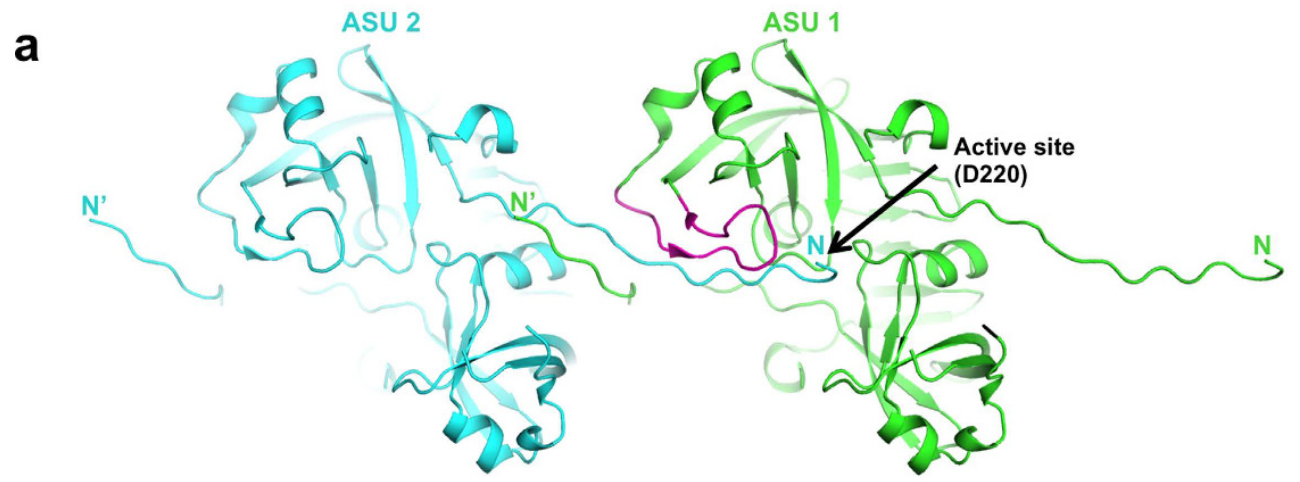

b

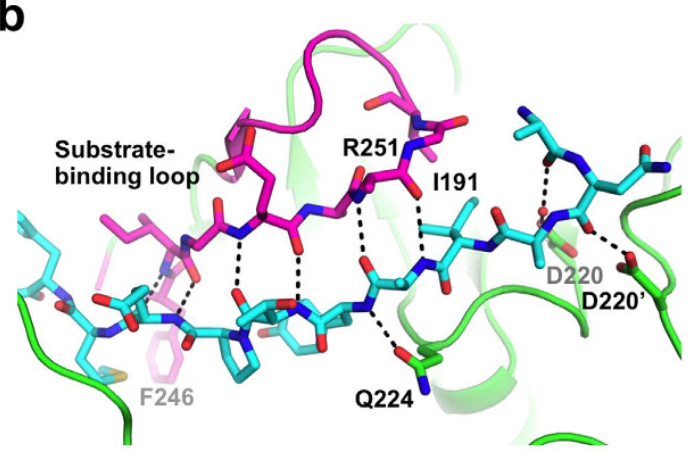

d

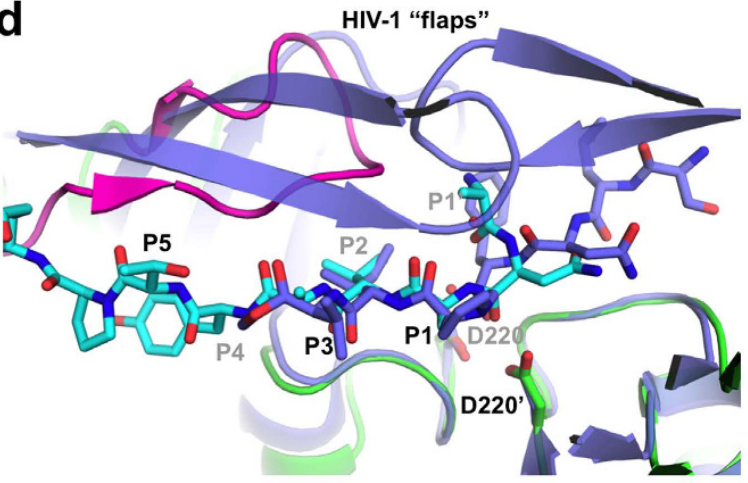

C

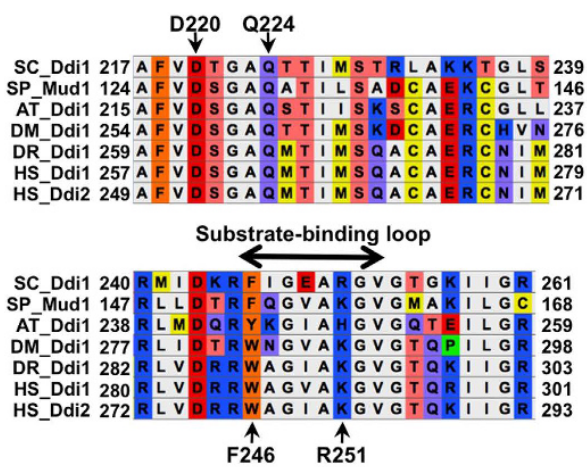

e

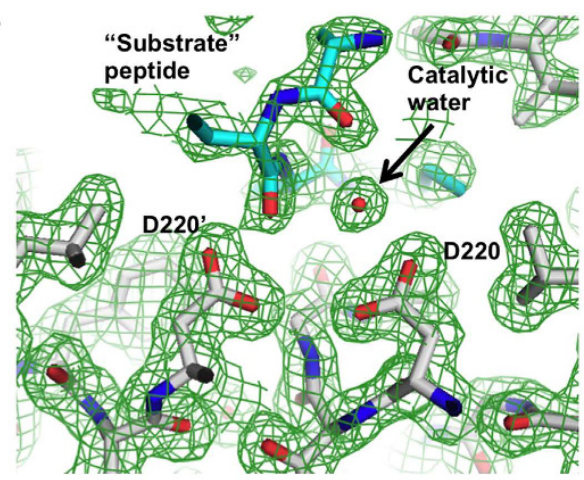

Figure 1. Crystal structure of the yeast Ddil protease domain reveals a potential substrate-binding mode. (a) Cartoon representation of two adjacent asymmetric units (ASU), showing the $\mathrm{N}$-terminus of one molecule in ASU \#2 (cyan) binding to the active site of a dimeric protease in ASU \#1 (green). The loop that forms interactions with the $\mathrm{N}$-terminal peptide is colored magenta. (b) Close-up view of the interaction between the $\mathrm{N}$-terminal peptide and the active site. Hydrogen bonds are shown as dashed lines, and important residues are labeled. (c) Sequence alignment of Ddil orthologs from different species. SC, Saccharomyces cerevisiae; SP, Schizosaccharomyces pombe; AT, Arabidopsis thaliana; DM, Drosophila melanogaster; DR, Danio rerio; HS, Homo sapiens. (d) Superposition of the Ddil protease structure (green) with HIV-1 protease bound to a peptide substrate mimetic (violet, PDB 7HVP). The HIV substrate mimetic is shown in blue, and the Ddil pseudosubstrate $\mathrm{N}$-terminal peptide is shown in cyan. (e) $2 F_{o}-F_{c}$ electron density maps of the active site, revealing the position of a water molecule that could act as a potential nucleophile in a proteolytic reaction.

Comparison with an HIV protease structure reveals substantial differences in substrate binding. In the structure of the HIV protease bound to a substrate-based hydroxyethylamine inhibitor ${ }^{32}$, two flaps wrap around the substrate analog (Fig. 1d). Main-chain amides in the flaps of HIV-1 protease form hydrogen bonds with a water molecule that also binds the substrate analog. In Ddil, the flap does not wrap around the pseudo-substrate, thus leaving it solvent-exposed. Remarkably, the pseudo-substrate and analogs in both Ddil and HIV-1 adopt very similar conformations, with main-chain atoms in the same configuration for amino acids in the P1 to P3 positions. Notably, both Ddil and HIV-1 protease substrates have an isoleucine in the P2 position. Overall, this suggests that the catalytic mechanism employed by both enzymes is similar, although they may engage their substrates differently.

The structure also reveals how Ddil could cleave a peptide bond: the catalytic residue Asp220 holds an ordered water molecule in place, which may act as the nucleophile for peptide bond hydrolysis (Fig. 1e). The symmetry-related Asp220' forms a hydrogen bond with a carbonyl in the P1' position, rendering it more 

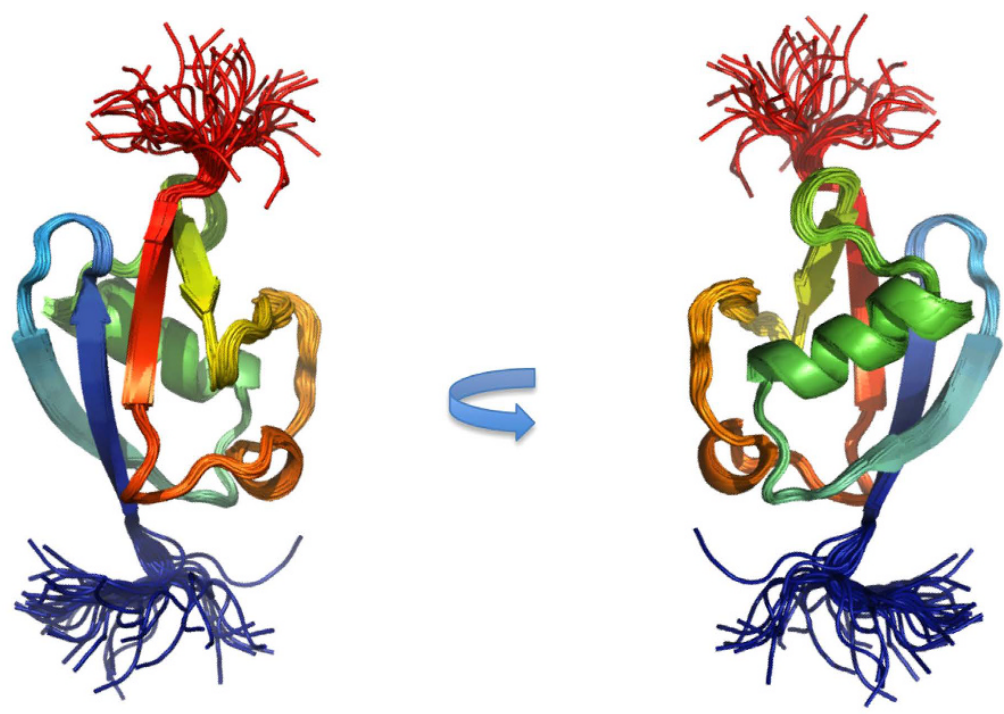

b

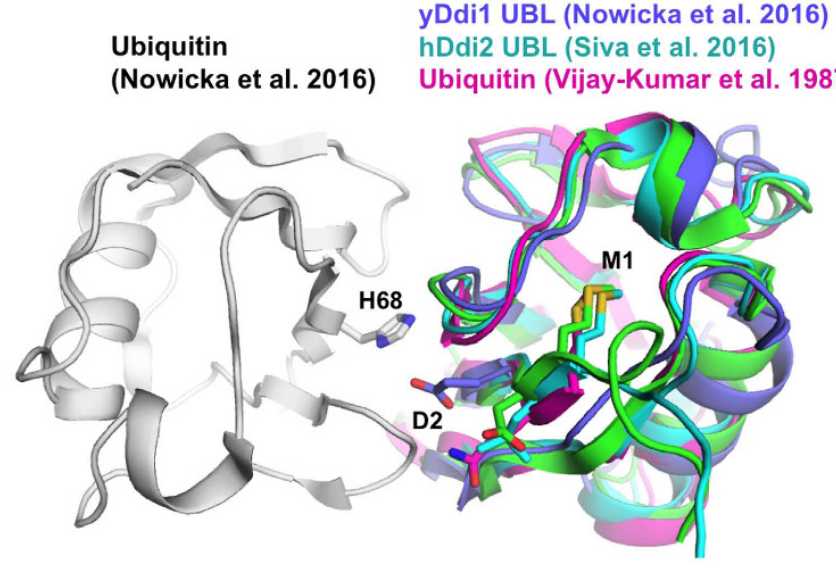

yDdi1 UBL (this work) yDdi1 UBL (Nowicka et al. 2016) hDdi2 UBL (Siva et al. 2016)

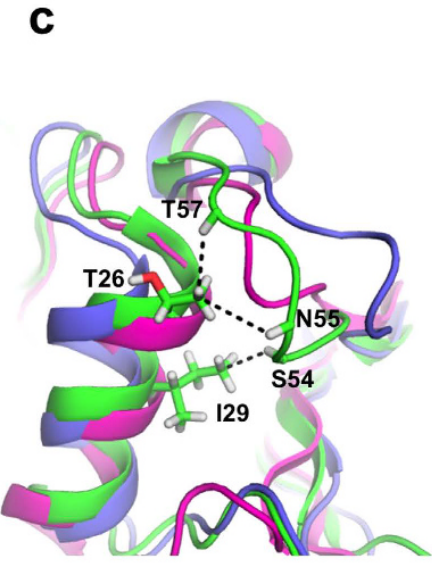

Figure 2. Solution structure of the Ddil UBL. (a) NMR solution structure of the UBL domain (a.a. 1-80; PDB 2N7E). An ensemble of 43 models is shown in cartoon representation, colored from blue to red from the N- to C-terminus. (b) Superposition of the yeast Ddi1 UBL NMR structure (green, pdb 2N7E) with the yeast Ddi1 UBL docked to ubiquitin (violet and white, pdb 2MWS), the human Ddi2 UBL NMR structure (cyan, pdb 2N7D), as well as the ubiquitin crystal structure (magenta, pdb 1UBQ). The side-chains of Met1 and Asp2 in the yDdi1 UBL (Gln2 and Leu2 in ubiquitin and hDdi2, respectively), and His68 in ubiquitin are shown as sticks. (c) Same as in (b), in a different orientation. The dashed lines indicate NOEs between $\mathrm{H} \alpha$ and methyl protons in the yeast Ddil UBL that confirms the proximity between the loop formed by a.a. $52-58$ and the N-terminal segment of an a-helix (a.a. 26-29).

susceptible to nucleophilic attack. The water molecule is within hydrogen-bonding distance of two carbonyl oxygens in the N-terminal fragment, but it is not positioned in a way that would enable catalytic attack of the carbonyl carbon. Thus, the observed conformation would not lead to proteolysis. Thus, it remains unknown how the protease activity can be triggered and what the substrate(s) might be.

Proteomics screen for substrate(s) of Ddi1 protease. To characterize putative substrate(s) of Ddil RVP, we used a proteomic technique that employs a proteome-derived peptide library as a proteolytic substrate screen $^{31}$. We used a peptide library derived from haploid yeast cells. We analyzed full-length Ddil as well as its "inactive" D220A variant at $\mathrm{pH} 4.0,5.0$, and 7.4. The data analysis revealed no Ddil-dependent cleavage at all pH tested, whereas the HIV-1 positive control produced significant amount of proteolysis (Supplementary Fig. S2). This suggests that the protease domain of Ddi1 requires activation or may cleave intact proteins in their native conformations.

The UBL and UBA domains of Ddi1 bind to ubiquitin. UBL domains are known to be protein:protein interaction modules, and thus could potentially play a role in protease substrate recognition. We thus characterized the structure and interactions mediated by the Ddil UBL domain using NMR. The domain adopts the ubiquitin fold (Fig. 2a,b and Supplementary Table 2) in spite of its low sequence similarity to ubiquitin. Yeast Ddil UBL has a rather shallow hydrophobic patch that is located at the same sequential and structural location as in human $\operatorname{Rad} 23 \mathrm{~A}^{33}$. This $\beta$-sheet patch potentially may be a protein-protein interaction site. However, NMR 
a

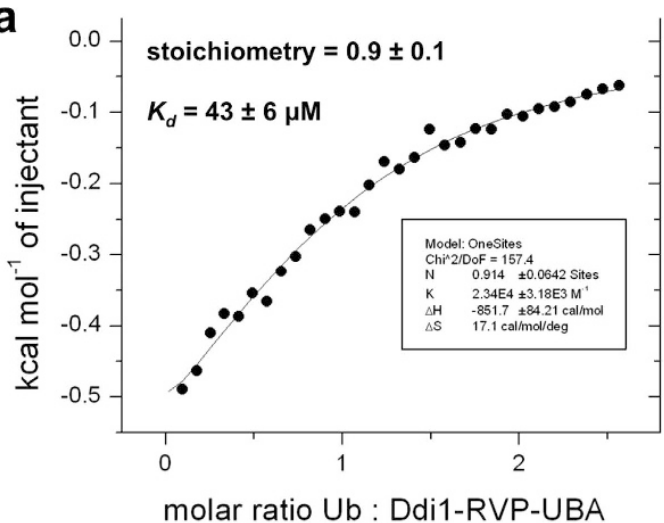

C

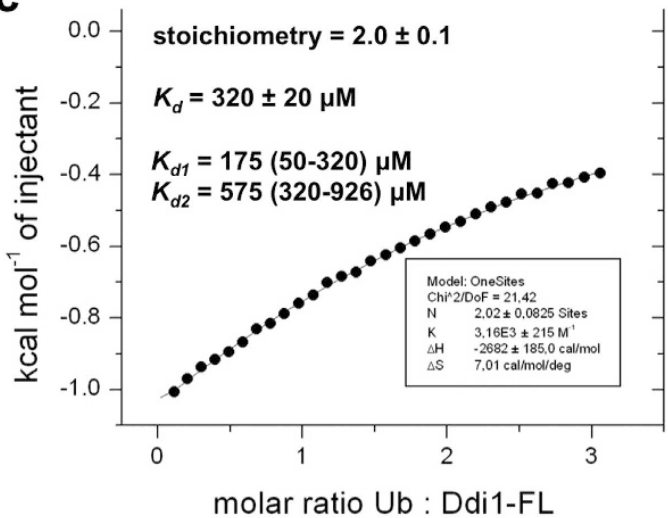

b

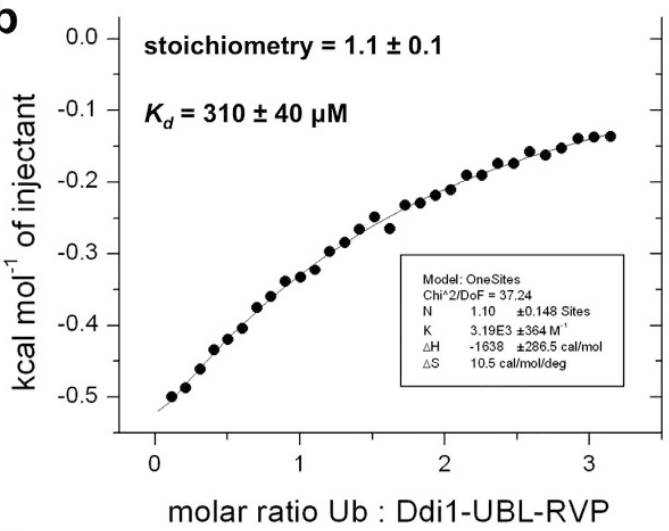

d

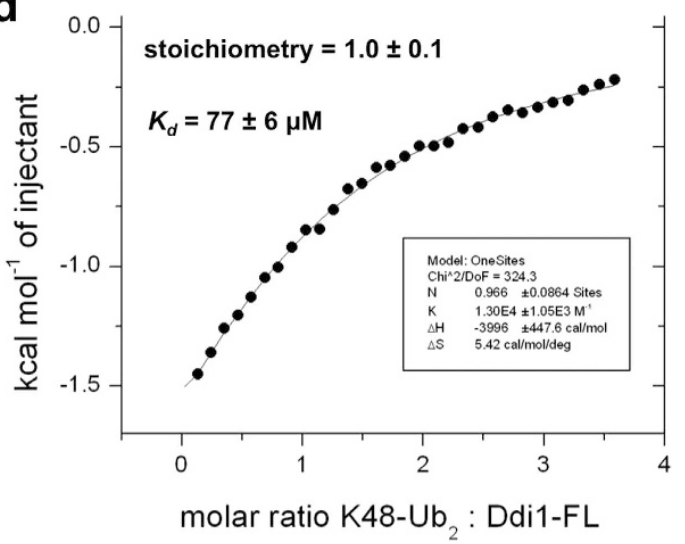

Figure 3. The UBL and UBA domains of Ddil bind to ubiquitin and diubiquitin. Ubiquitin or K48-linked diubiquitin $\left(\mathrm{Ub}_{2}\right)$ were added by syringe to Ddil constructs in the sample cell. The different titrations were (a) ubiquitin to RVP-UBA, (b) ubiquitin to UBL-RVP, (c) ubiquitin to full-length Ddi1, and (d) K48-Ub ${ }_{2}$ to full-length Ddi1. For full-length Ddi1 binding to ubiquitin (c), the data was also fitted to a model with two independent binding sites $\left(K_{d 1}\right.$ and $\left.K_{d 2}\right)$, each with a stoichiometry of 1 .

titrations of ${ }^{15} \mathrm{~N}$-Ddi1 UBL with potential UIM-containing ligands Ufo1 and Rpn10 showed no chemical shift perturbations, as reported by others (Supplementary Fig. S3) ${ }^{34,35}$. Moreover, no significant chemical shift perturbations were observed with the addition of Ddi1 86-325 (helical domains + protease) or Ddi1 388-428 (UBA), suggesting that the UBL would make no intramolecular contacts with other Ddil domains in the context of the full-length protein.

During preparation of this manuscript, the structure of Ddil UBL was published and its interaction with ubiquitin reported ${ }^{34}$. Comparison of the structures revealed small but significant differences. In our structure, the loop spanning a.a. $52-58$ is in proximity to the $\mathrm{N}$-terminus of the $\alpha$-helix formed by a.a $24-34$, with unambiguous NOEs between the two segments (Fig. 2c). This conformation, similar to the one found in ubiquitin, is different in the yeast Ddil UBL structure previously reported, where it is more distant from the $\alpha$-helix. Moreover, our construct includes the $\mathrm{N}$-terminal Met1 residue, which was absent from the construct used by Nowicka et al. The main-chain carbonyl of Met 1 makes a hydrogen bond with Val19 and extends the first $\beta$ strand, and its side-chain is oriented towards the core of the domain, as in ubiquitin or in the human Ddi2 UBL domain ${ }^{36}$ (Fig. 2b). This confers a different orientation to Asp2, which side-chain points towards His68 in ubiquitin docked to yeast Ddil $\mathrm{UBL}^{34}$.

To determine whether our Ddil constructs would bind to ubiquitin, we used isothermal titration calorimetry (ITC). We tested binding in the context of the full-length protein, with deletion of either the UBL or the UBA domain. Ubiquitin was found to bind the RVP-UBA construct (without the UBL) with a $K_{d}$ of $43 \mu \mathrm{M}$, whereas it bound the UBL-RVP construct (without the UBA) with a $K_{d}$ of $310 \mu \mathrm{M}$ (Fig. 3a,b). Titration of full-length Ddil with ubiquitin yielded an average $K_{d}$ of $320 \mu \mathrm{M}$, with a 2:1 stoichiometry (Fig. 3c). We also fitted the latter data to a model with two independent sites and found ranges of $K_{d}$ values that are close to the values obtained for the deletion constructs. These results are thus consistent with both UBA and UBL interacting with ubiquitin. Finally, we found that $\mathrm{K} 48-\mathrm{Ub}_{2}$ binds full-length Ddil with a $K_{d}$ of $77 \mu \mathrm{M}$ and a 1:1 stoichiometry, suggesting that each Ddil dimer binds two K48-Ub 2 molecules (Fig. 3d).

The structure of the helical domain of Ddi1 reveals similarities to DNA-binding domains. In an effort to elucidate the function of Ddil, we extended our structural studies beyond its RVP and UBL domains. As previously observed ${ }^{29}$, secondary structure prediction of Ddil shows that there is an $\alpha$-helical region juxtaposed to the N-terminus of the protease domain (Fig. 4a). This domain is separated from the UBL domain by a linker of variable length in different organisms, but is always juxtaposed to the RVP domain. To confirm that this region 
a

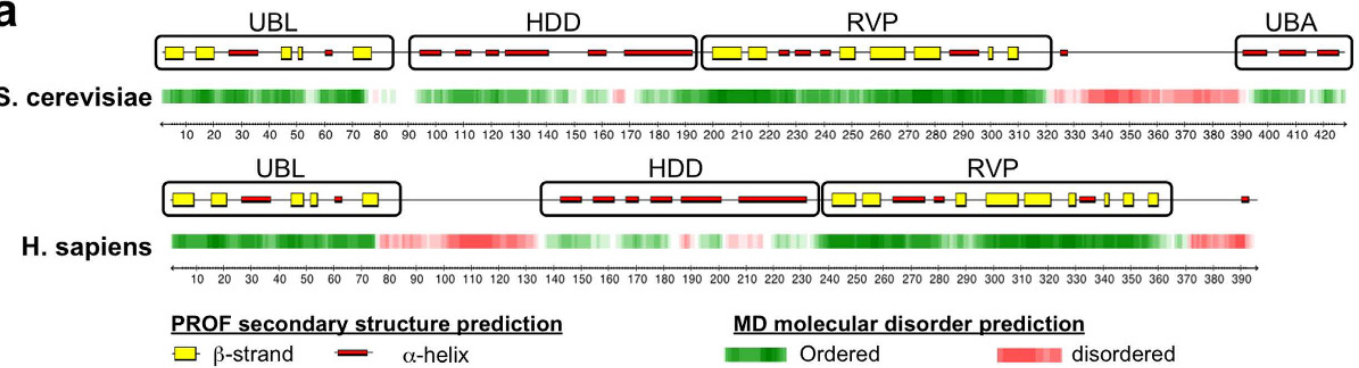

b
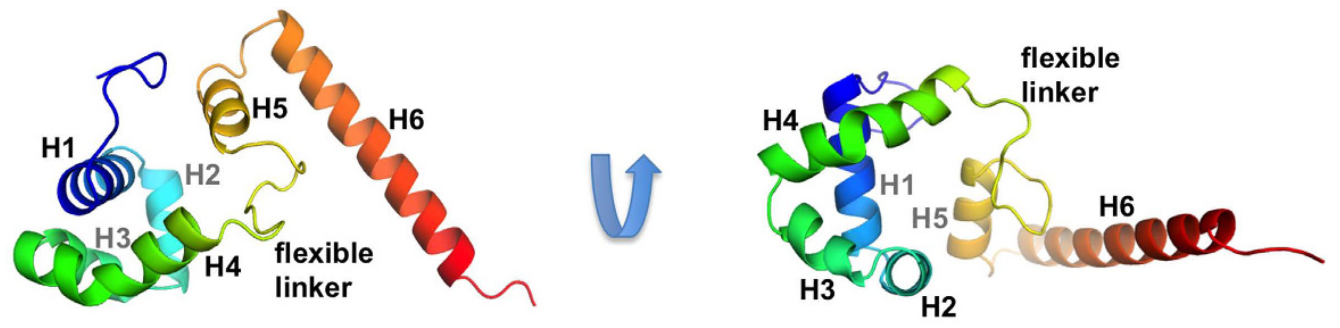

C
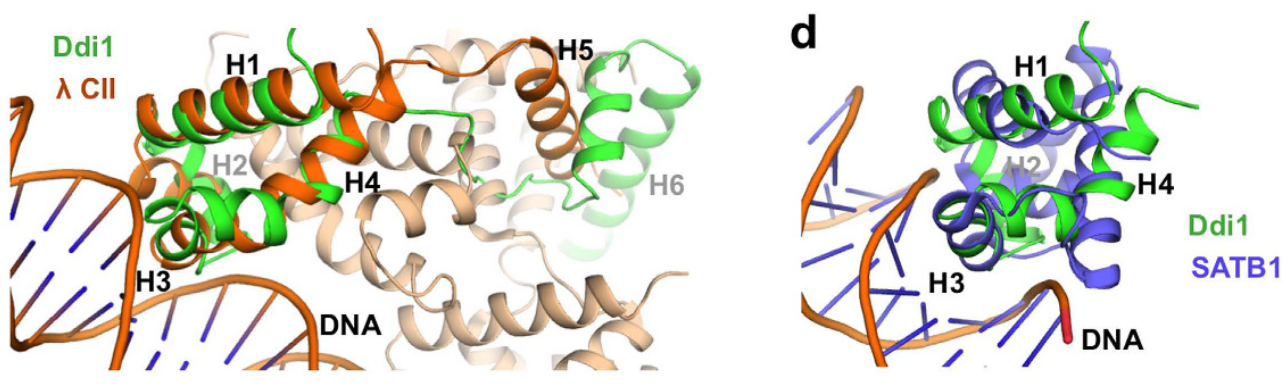

e
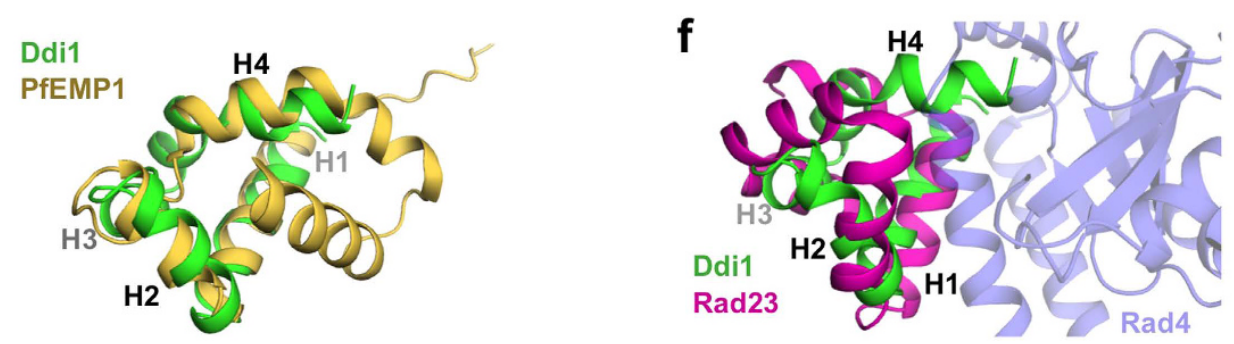

Figure 4. Ddil contains two helical domains preceding the protease domain. (a) Secondary structure and molecular disorder predictions of the yeast (top) and human (bottom) Ddil proteins. Predictions were performed on the PredictProtein server (http://www.predictprotein.org). (b) Cartoon of a representative model from the ensemble of HDD solution NMR structures, colored progressively from blue (N-terminus) to red (C-terminus). A flexible linker between helices 4-5 connects the two domains. (c-f) Structural superposition of the Ddil HDD N-terminal domain (green) with various homologous domains: (c) the bacteriophage $\lambda$ CII transcription activator bound to a DNA duplex (orange, PDB 1ZS4). Other protein chains in the $\lambda$ CII structure are colored in pale orange, showing the $5^{\text {th }}$ helix mediating tetramerization. (d) SATB1 CUT domain bound to a DNA duplex (blue, PDB 2O4A). (e) Intracellular domain of Plasmodium falciparum erythrocyte membrane protein 1 (yellow, PDB 2LKL). (f) Rad23 XPCB domain (magenta, PDB 2F4M) bound to Rad4 (pale blue).

effectively forms one or many folded domains, we expressed ${ }^{15} \mathrm{~N},{ }^{13} \mathrm{C}$-labeled Ddil (residues $\left.86-196\right)$ and characterized its structure by NMR. Its ${ }^{1} \mathrm{H},{ }^{15} \mathrm{~N}$ HSQC spectrum showed good signal dispersion in the proton dimension, indicating that the construct is folded (Supplementary Fig. S4a). We confirmed that this region of Ddil effectively adopts a folded structure using ${ }^{15} \mathrm{~N}-{ }^{1} \mathrm{H}$ heteronuclear NOE, which shows positive values except for the flexible $\mathrm{N}$ - and C-termini (Supplementary Fig. S4b). Because this helical region is folded and conserved across Ddi1 orthologs (Supplementary Fig. S4c), we named it the Helical Domain of Ddil (HDD).

The solution structure of HDD was determined using dihedral and NOE distance restraints, as well as residual dipolar couplings (RDCs) (Supplementary Table 3). The HDD actually consists of two alpha-helical domains (Fig. 4b): the N-terminal domain (residues 89-141) is a bundle of four helices with a hydrophobic core formed by some of the most conserved residues of the Ddil HDD. The C-terminal domain (residues 150-190) forms a hairpin with two helices, with a small hydrophobic core involving helix 1 and the first portion of the long helix 2 . The structure calculation converged for each domain, with backbone average pairwise rmsd of 0.57 and $0.99 \AA$ for the N-and 
C-terminal domains, respectively. However, there is considerable variability in the relative position of each domain (Supplementary Fig. S5a). A 10-residue linker with lower heteronuclear NOE values (Supplementary Fig. S4b) as well as low sequence conservation amongst Ddil orthologs (Supplementary Fig. S4c) connects the two parts of HDD. No long-range ${ }^{1} \mathrm{H}-{ }^{1} \mathrm{H}$ NOE was detected between the $\mathrm{N}$ - and $\mathrm{C}$-terminal domains, implying that they do not pack against each other. To determine the extent of the dynamic motion between the two domains, we analyzed small-angle X-ray scattering (SAXS) data using ensemble optimization ${ }^{37,38}$. The wide size and $R_{g}$ distributions of the optimized ensembles of tethered domains structures hint to a dynamic regime with a slightly more compact configuration than expected from a random pool of structures (Supplementary Fig. S6). Finally, the alignment tensor rhombicities are significantly different, further confirming they tumble independently from each other (Supplementary Fig. S5b,c).

To gain insight into the potential function of the HDD region of Ddil, we used the structure as a query to search for homologous domains in the Protein Data Bank (Supplementary Fig. S7a). Overall, we find that the HDD N-terminal domain (HDDnt) is similar to a wide and disparate set of alpha-helical bundle structures. However, the HDDnt displays striking similarity to DNA-binding domains from transcriptional regulators. Notably, the DNA-binding motif of the bacteriophage $\lambda$ cII transcription activator has a C $\alpha$ rmsd of $2.9 \AA$ with HDDnt (Fig. 4c). The domain is followed by a flexible tether and a long helix that can take multiple positions ${ }^{39}$, similar to the C-terminal domain of the HDD. This $\lambda$ cII C-terminal helix mediates tetramer formation upon DNA-binding. The HDDnt domain is also similar to the POU-specific OCT-1 DNA-binding domain (rmsd 3.9 $)$ ), as well as to the homologous CUT domain from human SATB1 (3.7 $\AA$, Fig. 4d). These DNA-binding domains are all four-helical bundles that insert helix 3 into the major groove and recognize specific DNA sequences (Fig. 4c,d). The fold search also revealed similarity between HDDnt and the intracellular domain of the erythrocyte membrane protein 1 from Plasmodium falciparum, but the latter is a five-helical bundle, and only the first four helices are similar to the HDDnt, suggesting their function are likely unrelated (Fig. 4e). Finally, weak sequence homology prompted us to compare the structure of HDDnt to helical bundles of the Stil-like family found in other UBL-UBA proteins such as Rad23 (Supplementary Fig. S7b). This domain binds to Rad4/XPC, a DNA-binding protein implicated in nucleotide excision repair. The first three helices of HDDnt are similar to XPC-binding motif of that $\operatorname{Rad} 23$ domain, but the $4^{\text {th }}$ helix adopts a completely different orientation (Fig. $4 \mathrm{f}$ ), suggesting it is unlikely that Ddil binds Rad4/XPC. Thus, the Ddi1 HDD is most similar to DNA-binding domains.

SAXS analysis suggests a dynamic structure for the Ddi1 dimer in solution. The Ddi1 protein consists of four domains with known structure, but how they are positioned to each other in the full-length protein is unclear. We therefore used SAXS to determine the relative position of the UBL and HDD domains with respect to the RVP dimer. Light scattering (dynamic and multiple-angle) was initially used to characterize and optimize solution scattering conditions for multiple Ddil constructs, except for the full-length protein, which formed aggregates that impeded analysis under all conditions tested. Constructs comprising the RVP domain (200-325) all formed dimers, as expected (Supplementary Fig. S8a). Ddil 86-325 slightly aggregated at pH 7, but increasing the $\mathrm{pH}$ and adding glycerol reduced the aggregation.

SAXS data from the RVP domain fit well to the dimeric crystal structure reported here, with a $\chi^{2}$ of 1.9 , confirming that the Ddi1 RVP adopts the same conformation in solution as observed in the crystal structure (Fig. 5a). SAXS data were also acquired on Ddi1 86-325 (HDD-RVP) and 2-325 (UBL-HDD-RVP). The $P(r)$ function reveals an asymmetrical pattern characteristic of elongated structures (Fig. $5 \mathrm{~b}$ ). To determine the relative positions of each domain, the SAXS data were fitted with the NMR structure of the UBL, the NMR structure of the HDD domain, and the crystal structure of the RVP domain as inputs. P2 symmetry was imposed, and the RVP domain position was kept constant. SAXS modeling of the Ddil HDD-RVP domains yielded two classes of structures, where the HDD domain extends on either side of the protease domain (Fig. 5c and Supplementary Fig. S8b). Calculations with the UBL-HDD-RVP data produced four classes of structures with similar overall shapes (Fig. 5c). The position of the UBL domain is highly variable and can be located on multiple sides of the HDD domain (Supplementary Fig. S8b). To determine the extent of the dynamics and reveal potential interactions among the domains, we also analyzed SAXS data using ensemble optimization ${ }^{37,38}$. In this approach, a pool of 10,000 models is generated from the structure of individual domains, connected by flexible linkers, to define the potential conformational space of the multi-domain protein. Then, a genetic algorithm is used to select a subset of conformers that best fit the experimental scattering data. In this case, P2 symmetry was maintained for the core RVP domain, but no symmetry was imposed on the UBL and HDD domains. Excellent fits were obtained for both data sets, and three independent calculations yielded similar $R_{g}$ and $D_{\max }$ distributions for the best-fit ensemble (Fig. 5d and Supplementary Fig. S9). The ensembles of HDD-RVP structures have more compact structures with smaller $R_{g}$ values than the pool (Fig. $5 \mathrm{~d}$ ). However analysis of $D_{\max }$ showed no clustering (Supplementary Fig. S9b), and the quantity $R_{f l e x}$ is close to that of the pool, suggesting the protein is flexible. Similar results were obtained for the UBL-HDD-RVP construct, albeit with a class of relatively compact structures dominating the ensemble (Fig. 5d). However, an overlay of these compact structures revealed no favored arrangement, and $R_{\text {flex }}$ is also high, reflecting flexibility. Overall, our analysis suggests that the Ddil protease dimer is flanked by flexible UBL and HDD domains that are in dynamic exchange in solution, albeit with a tendency towards more compact configurations.

\section{Discussion}

The focus of this study was to investigate the structure of yeast Ddil and the interactions mediated by its UBL domain. Surprisingly, we found that the UBL was unable to bind any of the UIMs that we tested, including all four UIMs found in the Ufo1 protein. Similar results were obtained by Nowicka et al. ${ }^{34}$. Based on the latter publication, we confirmed that the yeast UBL domain binds to ubiquitin. However, our ITC-based affinity measurements deviate from the reported affinities measured by NMR (150 and $45 \mu \mathrm{M}$ for the UBA and UBL, respectively ${ }^{34}$ ), 
a

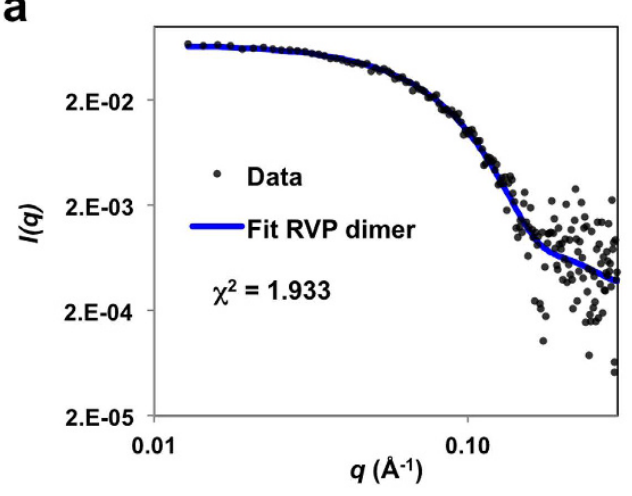

C

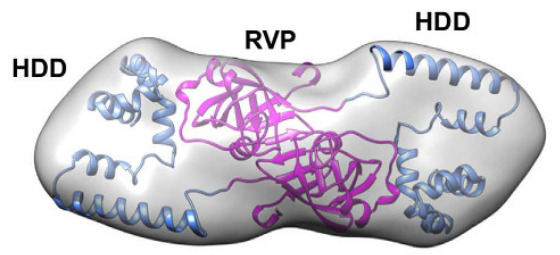

d

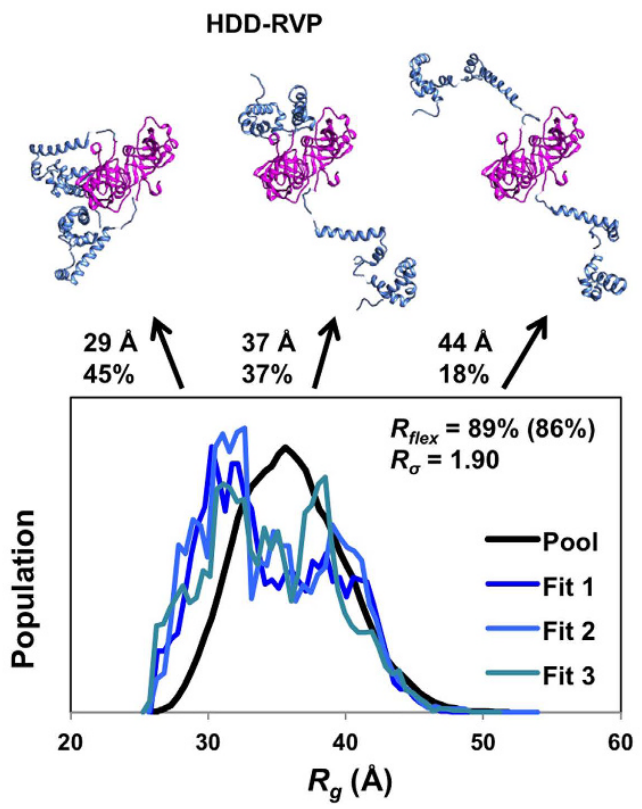

b
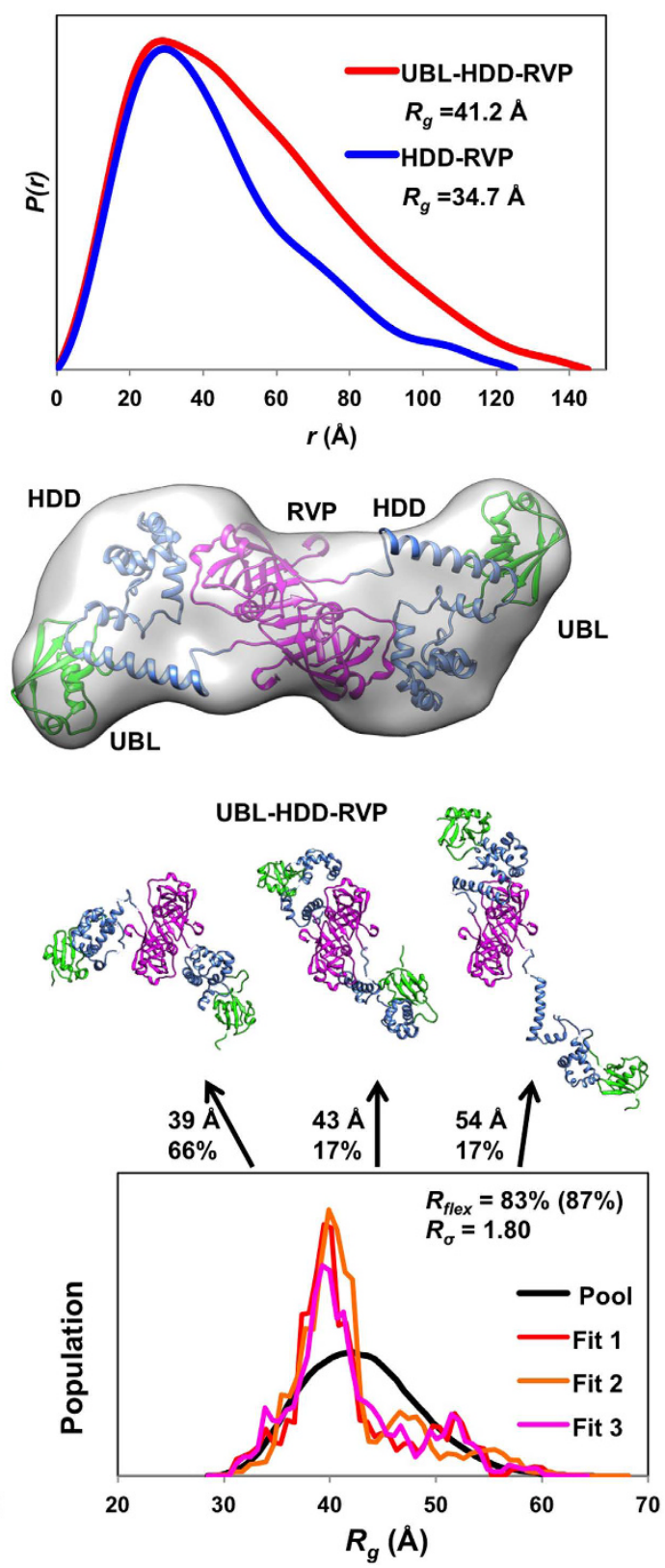

Figure 5. SAXS analysis of the Ddil dimer in solution. (a) SAXS data (circles) and calculated scattering curves (blue line) derived from the dimeric Ddi1 RVP domain crystal structure (200-325), displayed as a double-logarithmic plot. (b) SAXS pair-distance distribution functions for Ddi1 HDD-RVP (86-325) or UBL-HDD-RVP (2-325). (c) Modeling of SAXS data for HDD-RVP (left) and UBL-HDD-RVP (right), using the NMR structure of the UBL (green) and HDD (blue) domains, and the crystal structure of the dimeric protease domain (magenta). The surface represents the average of all models generated, contoured at $1.1 \times$ the volume of the particle. The cartoon displays a representative model from the ensemble. (d) Dynamic ensemble analysis of Ddi1 HDD-RVP (left) and UBL-HDD-RVP (right) using EOM. Each graph shows the distribution of $R_{g}$ for a pool of 10,000 structures randomly generated, and three sets of 100 ensembles that best fit the data. The structures of the best ensemble is shown on top, with $R_{g}$ and fraction indicated for each. These models suggest the behaviour of the flexible protein in solution, and does not represent the only solution.

which could be attributed to difference in buffers (sodium phosphate $\mathrm{pH} 6.8$ versus HEPES pH 7.4 here) and/or the use of different constructs. Indeed, the NMR titrations were carried out with isolated domains, as opposed to the ITC titrations that were performed in the context of the full-length dimeric protein. The association rate constants of protein:protein interactions is dependent on translational and rotational diffusion rates and protein electrostatics, which vary with protein size and depends on the context in which the domain is positioned ${ }^{40}$. These factors could explain the 3 -fold difference in $K_{d}$ (about $0.7 \mathrm{kcal} / \mathrm{mol}$ in free energy) we measured for the Ddi1 UBA domain. Moreover, the construct used by Nowicka et al. lacked the initiator Met1, which alters the 
position of Asp2, poised to interact with ubiquitin (Fig. 2b). This would reduce the affinity of our construct for ubiquitin, and we indeed observe a 6-fold difference. Whether the yeast Ddil protein retains its $\mathrm{N}$-terminal Met1 is unknown, but proteins with a charged residue in the second position are typically not excised, and often acetylated $^{41}$. Nevertheless, our data are overall consistent with yeast Ddil having two independent binding sites for ubiquitin. We also found that full-length yeast Ddil binds K48-Ub $\mathrm{U}_{2}$ with a 1:1 stoichiometry. These results could be explained by a mixture of binding modes, including binding to the UBL and UBA within the same Ddi1 molecule, or two UBL or two UBA domains in the dimer. The presence of two ubiquitin-binding domains in yeast Ddil may explain why some orthologs lack one or the other domain. For example, the S. pombe ortholog Mud1 does not have a UBL domain, but its UBA domain binds tightly to K48-Ub ${ }_{2}^{5}$. However, in an accompanying publication $^{36}$, we found that the UBL domain of human Ddi2, which lacks a UBA domain, does not bind to ubiquitin, implying that ubiquitin-binding is not a conserved attribute of the Ddil-like eukaryotic family of proteins.

Our crystal structure of the RVP domain reveals that the protease domain binds the N-terminus of the protein construct, which adopts an extended $\beta$ conformation through interaction with a conserved loop adjacent to the active site (Fig. 1). This loop normally forms a flap in aspartyl proteases of retroviruses such as HIV1 , but in our structure, the loop forms an extensive hydrogen-bonding network with the N-terminal segment pseudo-substrate. This conformation is likely a crystallization artefact for the following reasons: in the previous crystal structure of the yeast Ddi1 RVP domain ${ }^{30}$, the segment 180-198 is disordered and not observed in the active site; the construct that was crystallized is not cleaved in solution (Supplementary Fig. S1); the segment 185-191 is actually part of the HDD domain, which adopts an alpha-helical conformation in solution (Fig. 4). Yet, the pseudo-substrate N-terminal segment, adopts a conformation similar to HIV-1 protease peptide substrates. This suggests that the yeast Ddil RVP domain indeed functions as a hydrolytic enzyme against polypeptides, and that the observed conformation likely represents a model of substrate binding.

Unlike retroviral aspartyl proteases, which are able to cleave peptide substrates in vitro, Ddil did not exhibit any protease activity in our proteomic screen against a library of peptides derived from the yeast proteome (Supplementary Fig. S2). While this could mean that this domain might simply not be a protease, it could also be that the protease is activated only in the context of its interactions with another protein. The newly identified HDD domain could serve as a substrate anchor in this context (Fig. 4). Our SAXS data show that the HDD extends on either side of the RVP dimer and could thus serve as a landing platform for a substrate (Fig. 5c). We report a similar configuration in human Ddi $2^{36}$ where the HDD is juxtaposed to the protease dimer, suggesting a conserved functional relationship between the HDD and RVP domains. However, ensemble modeling of the SAXS data shows that the UBL and HDD domains are likely dynamic and can adopt multiple configurations (Fig. 5d). Considering the underdetermined nature of SAXS data, we cannot conclude whether the UBL-HDD-RVP module adopts a single rigid or multiple dynamic conformations. However, we note that NMR titrations with the UBL and the HDD-RVP constructs revealed no interaction, in agreement with the dynamic nature of the UBL-HDD-RVP module.

The role of the HDD remains unclear, but it is likely mediating interactions with a potential substrate of the Ddi1 protease. Stil-like domains homologous to the HDD have indeed been implicated in protein-protein interactions. In particular, the Rad23 Sti1-like domain forms a complex with the nucleotide excision repair protein $\operatorname{Rad} 4 / \mathrm{XPC}^{42,43}$, and the role of this interaction might be to protect Rad4/XPC from proteasomal degradation ${ }^{44}$. Dsk2/ubiquilin also contains a Sti1-like domain that has been proposed to bind the Hsp70-like protein Stch ${ }^{45}$. More strikingly, the structural homology with DNA-binding domains hints to the possibility that Ddil might be recognizing specific DNA motifs itself. The domain has some hallmarks of DNA-binding domains, such as a conserved basic residue (Arg131) that faces the DNA phosphate backbone in the structure alignment with the bacteriophage $\lambda$ CII transcription activator bound to a DNA duplex. This is consistent with the observation that Ddil localizes to the nucleus ${ }^{24}$. As Ddil is implicated in the DNA-damage response and cell cycle checkpoints ${ }^{11,15}$, it is possible that Ddi1 HDD binds to DNA damage sites whose regulation requires an ubiquitin-dependent proteolysis event. Future work should focus on identifying binding partners for Ddil HDD.

\section{Methods}

Protein expression and purification. The DDI1 gene was amplified by PCR from yeast genomic DNA and used as a template to generate fragments containing UBL (residues 2-80), HDD (86-196), RVP (185-325), HDD-RVP (86-325), and UBL-HDD-RVP (2-325). Yeast Ufo1 UIM1-4 (residues 512-668), UIM1 (512-539), UIM2 (542-569), and UIM3 (577-608) were similarly amplified by PCR. Rpn10 cloning was described previously $^{46}$. These fragments were cloned into the pGEX-6p1 plasmid in-frame with an N-terminal GST tag via $\mathrm{BamH} 1$ and $\mathrm{XhoI}$ sites. Constructs were expressed overnight with $0.5 \mathrm{mM}$ IPTG at $20^{\circ} \mathrm{C}$ in E. coli BL21(DE3) cells, resuspended in TBS buffer ( $50 \mathrm{mM}$ Tris- $\mathrm{HCl}, \mathrm{pH}$ 8.0, $100 \mathrm{mM} \mathrm{NaCl}, 5 \mathrm{mM} \beta$-mercaptoethanol) supplemented with $1 \mathrm{mM}$ EDTA, and lysed by sonication. The fusion protein was purified using glutathione-sepharose affinity and eluted with $20 \mathrm{mM}$ glutathione dissolved in TBS. The fusion protein was cleaved overnight at $4^{\circ} \mathrm{C}$ with the 3C protease and applied onto size-exclusion Superdex 200 or Superdex 75 16/600 chromatography columns (GE Healthcare). Contaminant GST was removed using glutathione-sepharose resin. Gel filtration was performed in NMR buffer (10 mM HEPES-NaOH, pH 7.0, $50 \mathrm{mM} \mathrm{NaCl}, 5 \mathrm{mM} \beta$-mercaptoethanol) for Ddil UBL, HDD, HDD-RVP, and UBA and Ufol UIM1-4, or SAXS buffer (10 mM Tris-HCl, pH 8.0, $50 \mathrm{mM} \mathrm{NaCl,} \mathrm{5 \%}$ glycerol, $5 \mathrm{mM} \beta$-mercaptoethanol) for constructs 2-325, 86-325, 185-325, and 86-196. Ufo1 UIM4 (651-668) was synthesized chemically. Single UIMs were further purified by C18 reverse chromatography and lyophilized prior to resuspension in NMR buffer.

Full-length yeast Ddi1, UBL (1-80), UBL-RVP (1-325), and RVP-UBA (180-428) were also cloned into pET16b vector (Novagen) in-frame with an $\mathrm{N}$-terminal histidine tag and used for ITC measurements. They were expressed in E. coli BL21(DE3)RIL host cells; subsequently resuspended in $50 \mathrm{mM}$ Tris- $\mathrm{HCl}, \mathrm{pH} 8.0,50 \mathrm{mM} \mathrm{NaCl}$, and $1 \mathrm{mM}$ EDTA; and lysed by three passages through an EmulsiFlex-C3 high pressure homogenizer (Avestin, 
Canada) at 1200 bar. Proteins were purified using nickel affinity chromatography and eluted with $250 \mathrm{mM}$ imidazole. Afterwards, they were dialyzed overnight into $50 \mathrm{mM}$ HEPES, pH 7.4, $150 \mathrm{mM} \mathrm{NaCl}$, and $10 \%$ glycerol and further purified by size-exclusion chromatography on a Superdex 200 16/60 gel filtration column (GE Healthcare). Individual fractions were analyzed by SDS-PAGE and/or Western blot.

The Ddil UBL with an N-terminal His-tag (residues $1-80$ ) was expressed as ${ }^{15} \mathrm{~N}$ - and ${ }^{15} \mathrm{~N} /{ }^{13} \mathrm{C}$-labeled proteins in cells grown in minimal medium containing $0.8 \mathrm{~g} / \mathrm{L}\left[{ }^{15} \mathrm{~N}\right]$ ammonium chloride and $2 \mathrm{~g} / \mathrm{L} \mathrm{d}-\left[{ }^{13} \mathrm{C}\right]$ glucose, as required. The purification procedure was the same as described above.

K48-linked $\mathrm{Ub}_{2}$ was synthesized using K48C and D77 ubiquitin mutants mixed with human E1 and yeast $\mathrm{Cdc} 34$, as previously described ${ }^{46,47}$. The products were purified by cation-exchange chromatography (mono S 5/50 GL, GE Healthcare) using $25 \mathrm{mM}$ sodium acetate, $\mathrm{pH} \mathrm{4.5}$, and $1 \mathrm{M} \mathrm{NaCl}$ for elution, and buffer-exchanged in ITC buffer.

X-ray crystallography. Ddi1 RVP (residues 185-325) was purified by gel filtration and concentrated to $8 \mathrm{mg} / \mathrm{mL}$ in $10 \mathrm{mM}$ Tris- $\mathrm{HCl}, \mathrm{pH} 7.4,50 \mathrm{mM} \mathrm{NaCl}$ and $1 \mathrm{mM}$ DTT. MALDI-TOF analysis revealed a single species with an average molecular weight of $16,320 \mathrm{Da}$ (predicted $16,313 \mathrm{Da}$ ). The protein was crystallized by vapor diffusion using the sitting drop technique by mixing $1 \mu \mathrm{L}$ of protein solution with $1 \mu \mathrm{L}$ of crystallization solution (0.1 M phosphate-citrate, $\mathrm{pH} 4.2,0.4 \mathrm{M} \mathrm{NaCl}, 20 \%$ PEG 8000). A crystal grew in 1-2 days. The crystal was cryo-protected by addition of $15 \%$ glycerol to the crystallization solution.

X-ray diffraction data at $100 \mathrm{~K}$ were acquired at the CHESS beamline Ala (Supplementary Table 1). A total of 240 images with an oscillation angle of 0.5 were collected. Reflections were integrated using iMOSFLM and scaled with SCALA as implemented in the CCP4 package ${ }^{48}$. The structure was determined by molecular replacement with the program Phaser $^{49}$, using chains A and B of the yeast Ddil RVP structure as a search model (PDB code $2 \mathrm{I}^{3} \mathrm{~A}^{30}$ ). Model building was performed using the program $\mathrm{COOT}^{50}$. Restrained and TLS refinement were performed using Refmac $5^{48}$.

Nuclear magnetic resonance spectroscopy. NMR spectra were acquired from $350 \mu \mathrm{l}$ samples of $0.2 \mathrm{mM}$ ${ }^{15} \mathrm{~N}$-labeled Ddi1 UBL for binding site mapping or $0.5 \mathrm{mM}{ }^{13} \mathrm{C} /{ }^{15} \mathrm{~N}$-labeled Ddi1 UBL for structural determination in a $50 \mathrm{mM}$ sodium phosphate buffer, $\mathrm{pH} 7.4$, containing $0.5 \%$ glycerol and $5 \% \mathrm{D}_{2} \mathrm{O} / 95 \% \mathrm{H}_{2} \mathrm{O}$. All NMR data for the UBL were collected at $25^{\circ} \mathrm{C}$ on a $600 \mathrm{MHz}$ Bruker Avance spectrometer equipped with a triple resonance $\left({ }^{15} \mathrm{~N} /{ }^{13} \mathrm{C} /{ }^{1} \mathrm{H}\right)$ cryoprobe. For determination of the sequence-specific resonance assignments for the UBL domain, a series of double and triple resonance spectra were collected as described previously $y^{51,52} .{ }^{1} \mathrm{H}-{ }^{1} \mathrm{H}$ distance constraints required to calculate the structure were derived from NOEs identified in $3 \mathrm{D}^{15} \mathrm{~N} /{ }^{1} \mathrm{H}$ NOESY-HSQC, and ${ }^{13} \mathrm{C} /{ }^{1} \mathrm{H}$ HSQC-NOESY spectra, which were acquired with an NOE mixing time of $120 \mathrm{~ms}$. Specific interaction of proteins and peptides with the Ddil UBL was monitored by changes induced in the positions of signals of ${ }^{15} \mathrm{~N}$-labeled Ddi1 UBL 2D ${ }^{15} \mathrm{~N} /{ }^{1} \mathrm{H}$ HSQC spectra using a recently described combined minimal shift approach ${ }^{53}$. A two-fold molar excess (0.4 mM) of Rpn10, Ddi1 HDD-RVP, Ddi1 UBA, Ufo1 UIM1-4, UIM1, UIM2, and UIM4 was added in these experiments. All spectra were processed using Topspin 3.2 (Bruker) and analyzed using Sparky (www.cgl.ucsf.edu/home/sparky).

All data sets for the Ddi1 86-196 HDD domain were acquired in HEPES-based NMR buffer at $30^{\circ} \mathrm{C}$ on a 600 or $850 \mathrm{MHz}$ Bruker NMR spectrometer both equipped with a triple-resonance $\left({ }^{1} \mathrm{H},{ }^{13} \mathrm{C},{ }^{15} \mathrm{~N}\right)$ cryoprobe. Heteronuclear ${ }^{1} \mathrm{H}_{-}{ }^{15} \mathrm{~N}$ NOE values were measured at $600 \mathrm{MHz}$, as described ${ }^{54} \cdot{ }^{15} \mathrm{~N}-{ }^{1} \mathrm{H}$ residual dipolar couplings were measured in $10 \mathrm{mg} / \mathrm{mL}$ Pf1 bacteriophage ${ }^{55}$ at $600 \mathrm{MHz}$ using a sensitivity-enhanced HSQC-IPAP experiment ${ }^{56}$. Backbone assignments were performed on ${ }^{15} \mathrm{~N},{ }^{13} \mathrm{C}$-labeled protein samples $(0.5 \mathrm{mM})$ using CBCACONH and HNCACB NMR experiments. ${ }^{1} \mathrm{H}-{ }^{1} \mathrm{H}$ distance constraints required to calculate the structure were derived from NOEs identified in $3 \mathrm{D}^{15} \mathrm{~N} /{ }^{1} \mathrm{H}$ NOESY-HSQC, and ${ }^{13} \mathrm{C} /{ }^{1} \mathrm{H}$ HSQC-NOESY spectra, which were acquired at $850 \mathrm{MHz}$ with an NOE mixing time of $120 \mathrm{~ms}$.

The family of converged structures for Ddil UBL and HDD were initially calculated using Cyana 2.1 ${ }^{57,58}$. NOE-derived restraints from $3 \mathrm{D}^{15} \mathrm{~N}$ - and ${ }^{13} \mathrm{C}$-edited NOESY spectra, which were assigned using combined automated NOE assignment and structure determination protocol, were used to produce preliminary structures. Backbone torsion angle constrains were generated from assigned chemical shifts using the program TALOS $+{ }^{59}$. For the UBL, hydrogen bond constraints involving residues with slowly exchanging amide protons were used in the calculations. Subsequently, five cycles of simulated annealing combined with redundant dihedral angle constrains were performed to produce a set of 43 converged structures with the lowest Cyana target function, no distance constraint violation and van der Waals violations greater than $0.2 \AA$, and no dihedral angle constraint violation greater than $5^{\circ}$. These were further refined in explicit solvent using the YASARA forcefield ${ }^{60}$. The structure of the HDD domain was further refined in XPLOR-NIH to incorporate residual dipolar couplings for residues displaying heteronuclear NOE values above 0.6, i.e. 90-141 (N-terminal domain) and 151-187 (C-terminal domain). Initial estimates and Monte Carlo calculations of the alignment tensor $D_{a}$ and $D_{r}$ were obtained using the software MODULE ${ }^{61}$. Satisfactory $R_{d i p}$ values of $30 \%$ and $35 \%$ were obtained for the N- and C-terminal domains, respectively, prior to the RDC refinement in XPLOR-NIH, indicating the accuracy of the NOE-derived structure. $D_{a}$ and $D_{r}$ were then optimized using a grid-search in XPLOR-NIH. Structures with the lowest total energy were selected.

Small-angle X-ray scattering (SAXS). Small-angle X-ray scattering data sets were collected on an in-house Anton Paar SAXSess camera equipped with a PANalytical PW3830 X-ray generator and a Roper/Princeton CCD detector. The beam length was set to $18 \mathrm{~mm}$, and the beam profile was recorded using an image plate for subsequent desmearing. Scattering data were collected at $4^{\circ} \mathrm{C}$ at protein concentrations of 4.0 and $8.0 \mathrm{mg} / \mathrm{mL}$ for 1 hour for Ddi1 $185-325,5.0$ and $10.0 \mathrm{mg} / \mathrm{mL}$ for 2 hours for $2-325,5.0$ and $10.0 \mathrm{mg} / \mathrm{mL}$ for 2 hours for $86-325$, and 5.0 and $10.0 \mathrm{mg} / \mathrm{mL}$ for 2 hours for $86-196$. Background scattering from the SAXS buffer was 
measured for 2 hours. Dark current correction, scaling, buffer subtraction, binning, desmearing, and merging were performed using SAXSquant 3.0 (Anton Paar). The merged scattering curves were then analyzed with different software included in the ATSAS package ${ }^{62}$. Scattering data were fitted to chains A \& B of the Ddil RVP crystal structure using CRYSOL, pair-distance distributions and $R_{g}$ values were calculated using GNOM. Molecular weights were estimated using the $Q_{r}$ invariant as described ${ }^{63}$. Ensemble optimization of the Ddi1 86-196 structure against the SAXS data was performed using EOM 2.0 by generating 10,000 structures with the NMR structures of the N-terminal (a.a. 89-141) and C-terminal (a.a. 150-191) HDD domains, selected using a genetic algorithm. Modeling of data collected from Ddi1 2-325 and 86-325 was performed using CORAL with the NMR structure of the UBL (a.a. 2-75), the two HDD domains (a.a. 89-141 \& 150-191) and the dimeric crystal structure of the RVP (a.a. 200-325). Twenty models with $\chi^{2}<1.6$ were generated and averaged using DAMAVER, with average $\chi^{2}$ of 1.36 and 1.19 for HDD-RVP and UBL-HDD-RVP, respectively. The resulting coordinates were used to generate pseudo-densities using Situs-pdb2 $\mathrm{vol}^{64}$ and contoured $10 \%$ above the particle volume derived from the Porod invariant (109,000 and $148,000 \AA^{3}$ for HDD-RVP and UBL-HDD-RVP, respectively) using UCSF-Chimera ${ }^{65}$. EOM 2.0 was used to generate 10,000 structures using the same domains as used in CORAL, with P2 symmetry imposed only on the RVP domain, using the genetic algorithm for conformer selection. The genetic algorithm was performed 100 times thrice to estimate the variability in the distribution of $D_{\max }$ and $R_{g}$ values.

Isothermal titration calorimetry (ITC). All calorimetric titrations of ubiquitin with full-length yeast Ddi1 and truncated variants were performed in $50 \mathrm{mM}$ HEPES, pH 7.4, $150 \mathrm{mM} \mathrm{NaCl}$ at $25^{\circ} \mathrm{C}$ using a VP-ITC system (MicroCal, GE Healthcare Life Sciences). For full-length Ddi1, $9 \mu \mathrm{L}$ aliquots of $1.42 \mathrm{mM}$ bovine ubiquitin (Sigma, cat. no. U6253) were injected stepwise into a sample cell containing $1.43 \mathrm{ml}$ of $97 \mu \mathrm{M}$ Ddil protein (concentration calculated to monomer). For UBL-RVP, $9 \mu \mathrm{l}$ aliquots of $2 \mathrm{mM}$ bovine ubiquitin were injected stepwise into a sample cell containing $1.43 \mathrm{ml}$ of $133.1 \mu \mathrm{M}$ Ddi1 UBL-RVP protein, and for RVP-UBA, $9 \mu \mathrm{l}$ aliquots of $796 \mu \mathrm{M}$ bovine ubiquitin were injected stepwise into a sample cell containing $1.43 \mathrm{ml}$ of $64.8 \mu \mathrm{M}$ Ddil RVP-UBA protein. The control dilution experiment, in which ubiquitin was injected into buffer alone, was also performed. All proteins used for titrations were properly dialyzed against buffer at $4{ }^{\circ} \mathrm{C}$ overnight, and their exact concentrations were determined by HPLC amino acid analysis. Titration of $\mathrm{K} 48-\mathrm{Ub}_{2}$ with full-length yeast Ddil was performed in $50 \mathrm{mM}$ HEPES, pH 7.4, $150 \mathrm{mM} \mathrm{NaCl}$ at $25^{\circ} \mathrm{C}$. Nine-microliter aliquots of $833.5 \mu \mathrm{M} \mathrm{K} 48-\mathrm{Ub}_{2}$ were injected stepwise into a sample cell containing $1.43 \mathrm{ml}$ of $48.5 \mu \mathrm{M}$ Ddil protein. Data sets were analyzed using Origin, using a one-site model by varying $N, K_{d}$ and $\Delta H$. For the titration with Ddil FL and ubiquitin, the data were also fitted to a two-sites model, where $N$ was fixed to 1.0 and $K_{d}$ and $\Delta H$ were floating variables for both sites. The range of values was determined by allowing the $\chi^{2}$ value to increase up to 37.3 , observed at $K_{d 1}=50 \mu \mathrm{M}$ and $K_{d 2}=926 \mu \mathrm{M}$, which still gives a satisfactory fit. The minimum $\chi^{2}$ value of 21.4 was observed at $K_{d 1}=175 \mu \mathrm{M}$ and $K_{d 2}=575 \mu \mathrm{M}$.

PICS assay and analysis. The PICS procedure was carried out as previously described ${ }^{31}$, and further details are included in our back-to-back publication ${ }^{34}$. Briefly, the amine-protected yeast proteome-derived peptide library $(1 \mathrm{mg} / \mathrm{ml})$ was incubated in $200 \mu \mathrm{L}$ buffer with $4 \mu \mathrm{g}$ of full-length yeast Ddi1 WT. The reaction was incubated for $12 \mathrm{~h}$ at $37^{\circ} \mathrm{C}$. The proteolytic cleavage assays were carried out in of $100 \mathrm{mM}$ sodium acetate, $300 \mathrm{mM} \mathrm{NaCl}, \mathrm{pH} 4.0,100 \mathrm{mM}$ sodium acetate, $300 \mathrm{mM} \mathrm{NaCl}, \mathrm{pH}$ 5.0, or $100 \mathrm{mM}$ HEPES, $300 \mathrm{mM} \mathrm{NaCl}, \mathrm{pH}$ 7.0. As negative controls, we used full-length Ddil with an inactivating mutation in its catalytic site (D220A), as well as a mock reaction with buffer. As a positive control, we tested the HIV-1 protease cleavage profile in $100 \mathrm{mM} \mathrm{Na}$ acetate, $300 \mathrm{mM} \mathrm{NaCl}, \mathrm{pH}$ 4.7, using wild-type and the catalytically inactive D25N mutant in a 1:200 protease-to-library ratio.

Data were analyzed by a series of predesigned queries in Microsoft Access database management software. First, lists of identified peptides from each MS run were filtered for peptides containing products of N-terminal modification by biotinylation. Second, peptides with over $80 \%$ confidence were picked for the tested enzyme, while peptides with over $10 \%$ confidence were picked for control reactions. To properly subtract the background signal, the list of peptides identified in the tested enzyme reaction was screened for peptides present in the mock reaction as well as in the reaction with catalytically inactive, and those peptides were removed from processing. Finally, peptides identified in the original unprocessed peptide library were removed from the analysis.

The final cleared list of identified peptides was then aligned with a FASTA proteomics database used for proteomics database search to determine the $\mathrm{N}$-terminal portions of cleaved peptides. If multiple computationally identified preceding sequences were found for one MS identified peptide, they were removed from processing, while the MS identified peptide sequences were kept in the list for downstream analysis. The final list of substrate peptides containing sequences of five P' amino acids identified in the MS experiment and five P amino acids identified computationally was then created. The frequency of each amino acid in each particular position was calculated and plotted, yielding the substrate specificity matrix.

\section{References}

1. Hershko, A. \& Ciechanover, A. The ubiquitin system. Annu Rev Biochem 67, 425-479 (1998).

2. Hicke, L. et al. Ubiquitin-binding domains. Nat Rev Mol Cell Biol 6, 610-621 (2005)

3. Bertolaet, B. L. et al. UBA domains of DNA damage-inducible proteins interact with ubiquitin. Nat Struct Biol 8, 417-422 (2001).

4. Wilkinson, C. R. et al. Proteins containing the UBA domain are able to bind to multi-ubiquitin chains. Nat Cell Biol 3, 939-943 (2001).

5. Trempe, J. F. et al. Mechanism of Lys48-linked polyubiquitin chain recognition by the Mud1 UBA domain. EMBO J 24, 3178-3189 (2005).

6. Raasi, S. et al. Diverse polyubiquitin interaction properties of ubiquitin-associated domains. Nat Struct Mol Biol 12, 708-714 (2005).

7. Elsasser, S. et al. Proteasome subunit Rpn1 binds ubiquitin-like protein domains. Nat Cell Biol 4, 725-730 (2002).

8. Saeki, Y. et al. Ubiquitin-like proteins and Rpn10 play cooperative roles in ubiquitin-dependent proteolysis. Biochem Biophys Res Commun 293, 986-992 (2002). 
9. Seeger, M. et al. Interaction of the anaphase-promoting complex/cyclosome and proteasome protein complexes with multiubiquitin chain-binding proteins. J Biol Chem 278, 16791-16796 (2003).

10. Gomez, T. A. et al. Identification of a functional docking site in the Rpn1 LRR domain for the UBA-UBL domain protein Ddi1. BMC Biol 9, 33 (2011).

11. Clarke, D. J. et al. Dosage suppressors of pds1 implicate ubiquitin-associated domains in checkpoint control. Mol Cell Biol 21, 1997-2007 (2001).

12. Diaz-Martinez, L. A. et al. Yeast UBL-UBA proteins have partially redundant functions in cell cycle control. Cell Div 1, 28 (2006).

13. Liu, Y. \& Xiao, W. Bidirectional regulation of two DNA-damage-inducible genes, MAG1 and DDI1, from Saccharomyces cerevisiae. Mol Microbiol 23, 777-789 (1997).

14. Liu, Y. et al. UAS(MAG1), a yeast cis-acting element that regulates the expression of MAG1, is located within the protein coding region of DDI1. Mol Gen Genet 255, 533-542 (1997)

15. Zhu, Y. \& Xiao, W. Differential regulation of two closely clustered yeast genes, MAG1 and DDI1, by cell-cycle checkpoints. Nucleic Acids Res 26, 5402-5408 (1998).

16. Zhu, Y. \& Xiao, W. Two alternative cell cycle checkpoint pathways differentially control DNA damage-dependent induction of MAG1 and DDI1 expression in yeast. Mol Genet Genomics 266, 436-444 (2001).

17. Fu, Y. et al. Rad6-Rad18 mediates a eukaryotic SOS response by ubiquitinating the 9-1-1 checkpoint clamp. Cell 133, 601-611 (2008).

18. Kaplun, L. et al. The DNA damage-inducible UbL-UbA protein Ddil participates in Mec1-mediated degradation of Ho endonuclease. Mol Cell Biol 25, 5355-5362 (2005).

19. Kaplun, L. et al. Functions of the DNA damage response pathway target Ho endonuclease of yeast for degradation via the ubiquitin26 S proteasome system. Proc Natl Acad Sci USA 97, 10077-10082 (2000).

20. Kaplun, L. et al. DNA damage response-mediated degradation of Ho endonuclease via the ubiquitin system involves its nuclear export. J Biol Chem 278, 48727-48734 (2003).

21. Ivantsiv, Y. et al. Unique role for the UbL-UbA protein Ddil in turnover of SCFUfo1 complexes. Mol Cell Biol 26, 1579-1588 (2006).

22. Lustgarten, V. \& Gerst, J. E. Yeast VSM1 encodes a v-SNARE binding protein that may act as a negative regulator of constitutive exocytosis. Mol Cell Biol 19, 4480-4494 (1999).

23. Marash, M. \& Gerst, J. E. Phosphorylation of the autoinhibitory domain of the Sso t-SNAREs promotes binding of the Vsm1 SNARE regulator in yeast. Mol Biol Cell 14, 3114-3125 (2003).

24. Gabriely, G. et al. Different domains of the UBL-UBA ubiquitin receptor, Ddi1/Vsm1, are involved in its multiple cellular roles. Mol Biol Cell 19, 3625-3637 (2008).

25. White, R. E. et al. The retroviral proteinase active site and the $\mathrm{N}$-terminus of Ddil are required for repression of protein secretion. FEBS Lett 585, 139-142 (2011).

26. Dixit, G. et al. Guanine nucleotide-binding protein (Galpha) endocytosis by a cascade of ubiquitin binding domain proteins is required for sustained morphogenesis and proper mating in yeast. I Biol Chem 289, 15052-15063 (2014).

27. Krylov, D. M. \& Koonin, E. V. A novel family of predicted retroviral-like aspartyl proteases with a possible key role in eukaryotic cell cycle control. Curr Biol 11, R584-R587 (2001).

28. White, R. E. et al. HIV proteinase inhibitors target the Ddil-like protein of Leishmania parasites. FASEB J (2011).

29. Perteguer, M. J. et al. Ddil-like protein from Leishmania major is an active aspartyl proteinase. Cell Stress Chaperones 18, 171-181 (2013)

30. Sirkis, R. et al. Ddil, a eukaryotic protein with the retroviral protease fold. J Mol Biol 364, 376-387 (2006).

31. Schilling, O. et al. Characterization of the prime and non-prime active site specificities of proteases by proteome-derived peptide libraries and tandem mass spectrometry. Nat Protoc 6, 111-120 (2011).

32. Swain, A. L. et al. X-ray crystallographic structure of a complex between a synthetic protease of human immunodeficiency virus 1 and a substrate-based hydroxyethylamine inhibitor. Proc Natl Acad Sci USA 87, 8805-8809 (1990).

33. Mueller, T. D. \& Feigon, J. Structural determinants for the binding of ubiquitin-like domains to the proteasome. EMBO J 22, 4634-4645 (2003).

34. Nowicka, U. et al. DNA-Damage-Inducible 1 Protein (Ddi1) Contains an Uncharacteristic Ubiquitin-like Domain that Binds Ubiquitin. Structure 23, 542-557 (2015).

35. Zhang, D. et al. Together, Rpn10 and Dsk2 can serve as a polyubiquitin chain-length sensor. Mol Cell 36, 1018-1033 (2009).

36. Siva, M. et al. Human DNA-Damage-Inducible 2 Protein Is Structurally and Functionally Distinct from Its Yeast Ortholog. Sci Rep 6, 30443 (2016).

37. Bernado, P. et al. Structural characterization of flexible proteins using small-angle X-ray scattering. J Am Chem Soc 129, 5656-5664 (2007).

38. Tria, G. et al. Advanced ensemble modelling of flexible macromolecules using X-ray solution scattering. IUCrJ 2, 207-217 (2015).

39. Jain, D. et al. Crystal structure of bacteriophage lambda cII and its DNA complex. Mol Cell 19, 259-269 (2005).

40. Schreiber, G. et al. Fundamental aspects of protein-protein association kinetics. Chem Rev 109, 839-860 (2009).

41. Bonissone, S. et al. $\mathrm{N}$-terminal protein processing: a comparative proteogenomic analysis. Mol Cell Proteomics 12, 14-28 (2013).

42. Min, J. H. \& Pavletich, N. P. Recognition of DNA damage by the Rad4 nucleotide excision repair protein. Nature 449, 570-575 (2007).

43. Masutani, C. et al. Identification and characterization of XPC-binding domain of hHR23B. Mol Cell Biol 17, 6915-6923 (1997).

44. Ng, J. M. et al. A novel regulation mechanism of DNA repair by damage-induced and RAD23-dependent stabilization of xeroderma pigmentosum group C protein. Genes Dev 17, 1630-1645 (2003).

45. Kaye, F. J. et al. A family of ubiquitin-like proteins binds the ATPase domain of Hsp70-like Stch. FEBS Lett 467, 348-355 (2000).

46. Riedinger, C. et al. Structure of Rpn10 and its interactions with polyubiquitin chains and the proteasome subunit Rpn12.J Biol Chem 285, 33992-34003 (2010)

47. Trempe, J. F. et al. A new crystal form of Lys48-linked diubiquitin. Acta Crystallogr Sect F Struct Biol Cryst Commun 66, 994-998 (2010)

48. Winn, M. D. et al. Overview of the CCP4 suite and current developments. Acta Crystallogr D Biol Crystallogr 67, 235-242 (2011)

49. McCoy, A. J. et al. Phaser crystallographic software. J Appl Crystallogr 40, 658-674 (2007).

50. Emsley, P. et al. Features and Development of Coot. Acta Crystallogr D Biol Crystallogr 66, 486-501 (2010).

51. Veverka, V. et al. NMR assignment of the mTOR domain responsible for rapamycin binding. J Biomol NMR 36 Suppl 1, 3 (2006).

52. Renshaw, P. S. et al. Sequence-specific assignment and secondary structure determination of the 195-residue complex formed by the Mycobacterium tuberculosis proteins CFP-10 and ESAT-6. J Biomol NMR 30, 225-226 (2004).

53. Veverka, V. et al. Structural characterization of the interaction of mTOR with phosphatidic acid and a novel class of inhibitor: compelling evidence for a central role of the FRB domain in small molecule-mediated regulation of mTOR. Oncogene 27, 585-595 (2008).

54. Kay, L. E. et al. Backbone dynamics of proteins as studied by $15 \mathrm{~N}$ inverse detected heteronuclear NMR spectroscopy: application to staphylococcal nuclease. Biochemistry 28, 8972-8979 (1989).

55. Hansen, M. R. et al. Tunable alignment of macromolecules by filamentous phage yields dipolar coupling interactions. Nat Struct Biol 5, 1065-1074 (1998). 
56. Cordier, F. et al. A doublet-separated sensitivity-enhanced HSQC for the determination of scalar and dipolar one-bond J-couplings. J Biomol NMR 13, 175-180 (1999).

57. Guntert, P. et al. Torsion angle dynamics for NMR structure calculation with the new program DYANA. J Mol Biol 273, 283-298 (1997).

58. Herrmann, T. et al. Protein NMR structure determination with automated NOE assignment using the new software CANDID and the torsion angle dynamics algorithm DYANA. J Mol Biol 319, 209-227 (2002).

59. Shen, Y. et al. TALOS+: a hybrid method for predicting protein backbone torsion angles from NMR chemical shifts. J Biomol NMR 44, 213-223 (2009).

60. Harjes, E. et al. GTP-Ras disrupts the intramolecular complex of C1 and RA domains of Nore1. Structure 14, 881-888 (2006).

61. Dosset, P. et al. A novel interactive tool for rigid-body modeling of multi-domain macromolecules using residual dipolar couplings. J Biomol NMR 20, 223-231 (2001)

62. Petoukhov, M. V. et al. New developments in the ATSAS program package for small-angle scattering data analysis. J Appl Cryst 45, 342-350 (2012).

63. Rambo, R. P. \& Tainer, J. A. Accurate assessment of mass, models and resolution by small-angle scattering. Nature 496, 477-481 (2013).

64. Wriggers, W. \& Chacon, P. Using Situs for the Registration of Protein Structures with Low-Resolution Bead Models from X-ray Solution Scattering. J Appl Cryst 34, 773-776 (2001).

65. Pettersen, E. F. et al. UCSF Chimera-a visualization system for exploratory research and analysis. J Comput Chem 25, 1605-1612 (2004).

\section{Acknowledgements}

We thank the staff at MacCHESS for assistance with X-ray data collection. This work was supported by a Canadian Institutes of Health Research (CIHR) postdoctoral fellowship and startup funds from McGill University and Fonds de recherche du Québec - Santé (FRQS) to J.-F.T., a CIHR grant to K.G. (MOP-14219). The McGill SPR-MS Facility thanks the Canada Foundation for Innovation (CFI) for infrastructure support. This work was supported by the Ministry of Education, Youth and Sports of the Czech Republic within the National Sustainability Program II (Project BIOCEV-FAR) LQ1604 and by the project "BIOCEV" (CZ.1.05/1.1.00/02.0109) and the Ministry of Education of the Czech Republic (program “NAVRAT” LK11205 and program "NPU I” LO1304).

\section{Author Contributions}

J.-F.T. conceived experiments, collected and analyzed crystallography, mass spectrometry, NMR and SAXS data, synthesized diubiquitin, wrote the manuscript, and prepared the figures. M.S., M.S., M.K., V.V., J.K. and K.G.Š. performed and analyzed the UBL structure determination and ITC experiments. C.D.H.R. performed NMR assignments on the HDD and purified the SAXS samples. A.H. performed NMR titrations with the UBL. M.M. performed molecular cloning. X.F. purified the RVP for crystallization. S.S. performed NMR assignments on the UBL. J.-F.T., K.G., J.K. and K.G.S. drafted the manuscript.

\section{Additional Information}

Accession Codes: Coordinates and structure factors for the RVP crystal structure were deposited in the PDB under accession code 4Z2Z. Coordinates and restraints for the Ddil UBL and HDD solution NMR structures were deposited in the PDB under accession codes 2N7E (BMRB code 25803) and 5KES (BMRB code 30102), respectively.

Supplementary information accompanies this paper at http://www.nature.com/srep

Competing financial interests: The authors declare no competing financial interests.

How to cite this article: Trempe, J.-F. et al. Structural studies of the yeast DNA damage-inducible protein Ddi1 reveal domain architecture of this eukaryotic protein family. Sci. Rep. 6, 33671; doi: 10.1038/srep33671 (2016).

This work is licensed under a Creative Commons Attribution 4.0 International License. The images or other third party material in this article are included in the article's Creative Commons license, unless indicated otherwise in the credit line; if the material is not included under the Creative Commons license, users will need to obtain permission from the license holder to reproduce the material. To view a copy of this license, visit http://creativecommons.org/licenses/by/4.0/

(C) The Author(s) 2016 